\title{
Albumin-Linked Doxorubicin (Aldoxorubicin) as Treatment for Relapsed Glioblastoma: A Case Report
}

Levitt D, Slim J, Jennifer N. Slim*, Boulmay B and Galliano G

Hematology and Oncology Service, Louisiana State University Health Science Center, New Orleans, LA, USA

"Corresponding author: Jennifer N. Slim, DO, Section of Hematology/Oncology, 533 Bolivar St. Room 526, New Orleans, La-70112, USA, Tel: (504) 568-2713; Fax: (504) 568-2127; E-mail: Jslim@Isuhsc.edu

Received date: Jan 30, 2015, Accepted date: Mar 12, 2015, Publication date: Mar 16, 2015

Copyright: (c) 2015 Levitt D, et al. This is an open-access article distributed under the terms of the Creative Commons Attribution License, which permits unrestricted use, distribution, and reproduction in any medium, provided the original author and source are credited.

\section{Introduction}

Glioblastoma multiforme (GBM) the most common primary brain malignancy in adults, has an approximately $25 \% 2$-year overall survival despite improvements in both first and second-line therapies [1]. However, options still remain limited in those patients progressing after failure of temozolomide. IDH mutation and MGMT methylation status do predict whether patients will respond favorable to alkylator based therapies and clinical trials are ongoing to develop therapies specific to these GBM subtypes. In many cases, chemotherapy resistance is thought to be related to the inability of agents to cross the blood brain barrier into the tumor, limiting efficacy of otherwise effective agents.

Aldoxorubicin is a novel cytotoxic agent currently undergoing trials in soft tissue sarcoma, small cell lung cancer and Kaposi's sarcoma. The drug consists of doxorubicin attached to a $\mathrm{pH}$-sensitive linker that binds covalently only to the cysteine- 34 amino acid in circulating albumin [2]. It has a half-life of approximately 20 hours, a low volume of distribution and clearance, and less than $2 \%$ free doxorubicin has been detected in the blood during a 72 hour testing period [3].

Marrero et al. demonstrated aldoxorubicin to be significantly more active than doxorubicin in a U87 human xenograft GBM model in mice [4]. Free doxorubicin administration appeared no better than saline and could not be detected in either the tumor or normal brain tissue. However, aldoxorubicin crossed the blood-brain barrier to enter the tumor but not normal brain parenchyma. Drug was concentrated in the tumor cell nucleus, significantly retarding tumor growth and resulted in increased survival [5].

An ongoing study is evaluating the preliminary safety and efficacy of administering aldoxorubicin to patients with recurrent glioblastoma (NCT02014844) [6]. We report here a patient who appeared to experience tumor progression after treatment with aldoxorubicin by both clinical and radiological assessments; however, pathological review post tumor debulking demonstrated no evidence of tumor. We would suggest that this likely represents pseudo-progression as can be seen in CNS malignancies undergoing radiation therapy, and may reflect the ability of albumin-bound doxorubicin (aldoxorubicin) to allow doxorubicin to enter glioblastoma and induce tumor necrosis.

\section{Case Report}

The patient is a 54 year old white man diagnosed with left parietal lobe GBM in January 2011. He underwent gross total surgical resection of the tumor and chemo-radiotherapy with temozolomide, followed by maintenance temozolomide. In April 2013 he received stereotactic radiosurgery for a small focus of tumor recurrence. The patient experienced a seizure in November 2013. MRI demonstrated edema and mass effect in the left parietal lobe area and he was treated with anti-convulsants and a dexamethasone taper. The mass effect increased in March 2014 and the patient had another debulking procedure in April 2014. Histopathology demonstrated radionecrosis but sizeable residual GBM tumor.

The patient was enrolled onto the aldoxorubicin trial and received a single cycle of intravenous aldoxorubicin, $350 \mathrm{mg} / \mathrm{m}^{2}\left(260 \mathrm{mg} / \mathrm{m}^{2}\right.$ doxorubicin equivalents)Treatment-associated adverse events included an episode of febrile neutropenia, grade 3 neutropenia and thrombocytopenia, and grade 2 mucositis, all of which resolved without sequelae. However, due to these adverse events the patient decided not to continue on aldoxorubicin treatment.

Brain MRI exams performed four and six weeks after therapy showed a mass effect due probably associated with edema. Radiology interpretation of imaging indicated a peripherally enhancing mass with central necrosis, consistent with GBM progression (Figure 1).

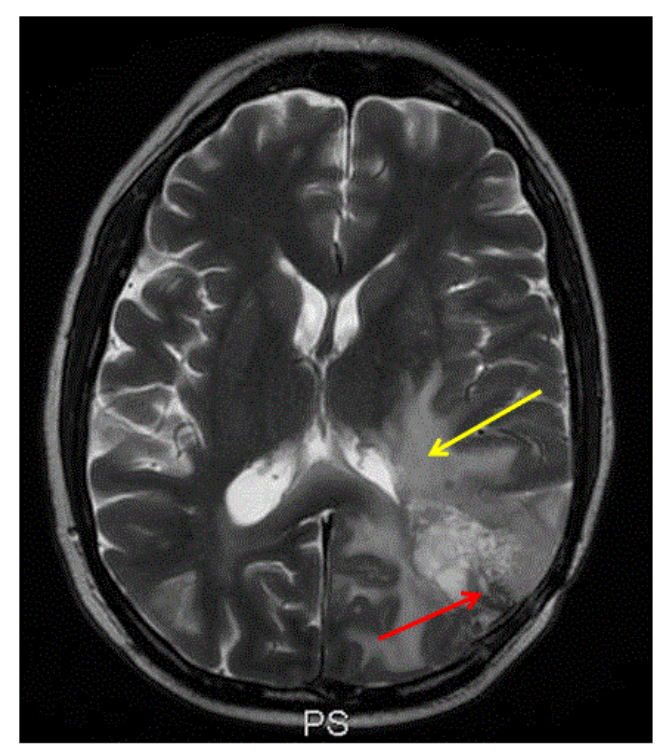

Figure 1: T2 weighted Brain MRI revealing High Grade Glioblastoma Multiforme with small necrotic area (red arrow) with associated edema (yellow arrow) and midline shift.

During this period the patient had progression of global weakness, fine motor tremor and did not recover his previous functionality. Eight weeks after the aldoxorubicin dose, the patient underwent resection of 
the left posterior temporal-parietal lobe lesion. He had received no other treatment for his tumor.

Histopathological assessment of the tissue showed predominantly therapy-related changes with areas of necrosis, fibrosis and hemosiderin deposition. There was no evidence of recurrent glioblastoma throughout the entire surgical specimen. Post-operative brain MRI showed decrease in size of the left temporal lobe tumor and the associated edema (Figure 2).

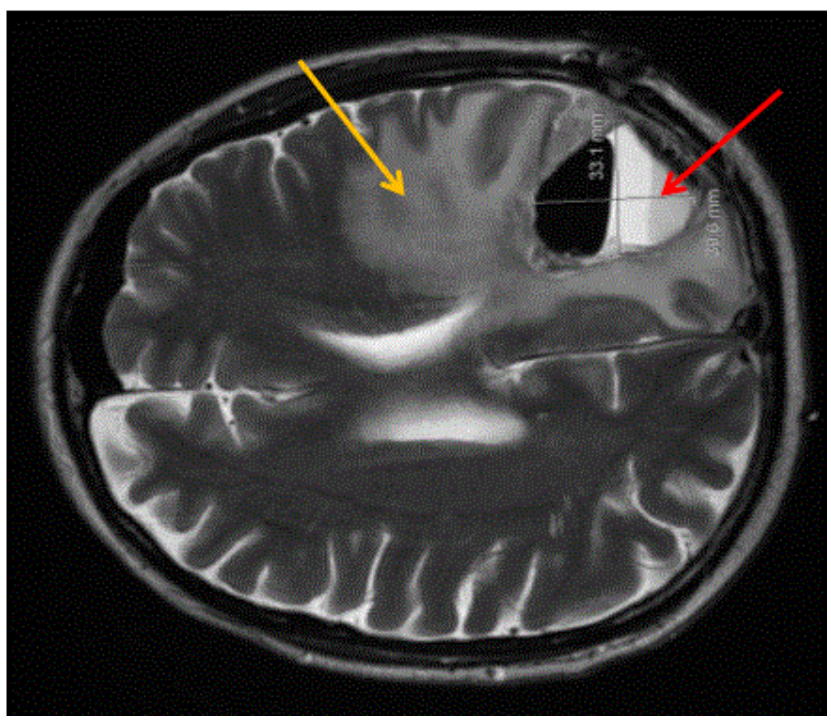

Figure 2: T2 weighted Brain MRI revealing High Grade Glioblastoma Multiforme with necrotic core (red arrow) due to outgrowing vascular supply with associated edema (yellow arrow) and midline shift.

\section{Discussion}

GBM is a lethal malignancy with few options to treat both primary and recurrent tumor. Doxorubicin is active in inducing glioblastoma cell death in vitro and can even be effective in treating systemicallyengrafted glioblastoma xenografts. Evidence suggests that free doxorubicin itself cannot cross even a disrupted blood brain barrier to enter a tumor, either in humans or rodents (Bigotte L, Arvidson B, Olsson Y. Cytofluorescense localization of Adriamycin in the nervous system, I: distribution of the drug in the central nervous system of normal adult mice after intravenous injection. Acta Neuropathologica (Berlin) 57: 121-129, 1982. Di Fronzo G, Lenaz L, Bonnadonna G. Distribution and excretion of Adriamycin in man. Biomedicine 19: 169-171, 1973. Von Holst H, Knochenhauer E, Blomgen $H$, et al. Uptake of Adriamycin in tumour and surrounding brain tissue in patients with malignant gliomas. Acta Neurochir 104: 13-16, 1990). Modified liposomal and nanoparticle doxorubicin using transferrin or folate, glutathione, tumor-penetrating peptides, cationic albumin, ondansetron pre-treatment or ultrasound to bypass the blood-brain barrier and be taken up into glioblastoma tumors transplanted into mouse brains. However, liposomal doxorubicin itself, either alone or in combination with temozolomide, does not appear to improve responses, PFS at 6 months, or survival (Ananda S, Nowak AK, Cher I, et al. Phase 2 trial of temozolomide and pegylated liposomal doxorubicin in the treatment of patients with glioblastoma multiforme following concurrent radiotherapy and chemotherapy. J Clin, Neurosci 18: 1444-1448, 2011).

A previous study in mice bearing orthotopically transplanted human glioblastoma cells in the brain demonstrated uptake of aldoxorubicin into tumor and tumor cell nuclei, but not normal brain parenchyma, with ensuing reduction in tumor growth, increased apoptosis and prolongation of survival by over $100 \%$. It is unclear why covalent binding to albumin allows doxorubicin to bypass drug resistant export pumps found at the blood brain barrier epithelium. Cationic albumin does allow pegylated nanoparticles to be taken up in the brain and aldoxorubicin, a doxorubicin-conjugated albumin, does seem to be moderately cationic (pKa 8.7) (Kremer P, Wunder A, Sinn $\mathrm{H}$, et al. Laser-induced fluorescence detection of malignant gliomas using fluorescein-labeled serum albumin: experimental and preliminary clinical results. Neurol Res. 22:481-9, 2000).

\section{Conclusion}

In this patient, despite radiological evidence of tumor progression, histopathological evaluation of debulked tumor showed no evidence of viable malignant tissue. However, this patient had completed treatment with radiation and temozolomide more than 2 years prior to being treated with aldoxorubicin, no other treatment for his malignancy was administered in the interim, and a debulking procedure a few months before he received aldoxorubicin demonstrated progressive tumor growth. It is feasible that appearance of tumor on the MRI scans post aldoxorubicin treatment was more likely pseudo-progression, a situation quite common in patients exposed to radiation plus temozolomide. Close monitoring and evaluation of scans in patients treated with aldoxorubicin will keep this issue at the forefront in the future. However, the likelihood that aldoxorubicin enables doxorubicin to enter and kill the tumor itself is both interesting and potentially important, and could add to the armamentarium of treatments for GBM.

\section{References}

1. Stupp R, Mason WP, van den Bent MJ, Weller M, Fisher B, et al. (2005) Tumor and Radiotherapy Groups and the National Cancer Institute of Canada Clinical Trials Group. Radiotherapy plus Concomitant and Adjuvant Temozolomide for Glioblastoma. N Engl J Med 352: 987-996.

2. Kratz F, Warnecke A, Scheuermann K, Stockmar C, Schwab J, et al. (2002) Probing the cysteine-34 position of endogenous serum albumin with thiolbinding doxorubicin derivatives. improved efficacy of an acid-sensitive doxorubicin derivative with specific albumin-binding properties compared to that of the parent compound. J Med Chem 45: 5523-5533.

3. Mita MM, Natale RB, Wolin EM, Laabs B, Dinh H, et al. (2014) Pharmacokinetic study of aldoxorubicin in patients with solid tumors. Invest New Drugs .

4. Marrero L, Wyczechowska D, Musto AE, Wilk A, Vashistha H, et al. (2014) Therapeutic efficacy of aldoxorubicin in an intracranial xenograft mouse model of human glioblastoma. Neoplasia 16: 874-882.

5. Hulsey KM, Mashimo T, Banerjee A, Soesbe TC, Spence JS, et al. (2014) H MRS characterization of neurochemical profiles in orthotopic mouse models of human brain tumors. NMR Biomed. 28: 108-115.

6. https://clinicaltrials.gov/ct2/show/NCT02014844. 